\title{
Micro-CT for Anatomic Referencing in PET and SPECT: Radiation Dose, Biologic Damage, and Image Quality
}

\author{
Veerle Kersemans ${ }^{1}$, James Thompson ${ }^{1}$, Bart Cornelissen ${ }^{1}$, Michael Woodcock ${ }^{1}$, Philip D. Allen ${ }^{1}$, Nico Buls ${ }^{2}$, \\ Ruth J Muschel ${ }^{1}$, Mark A. Hill ${ }^{1}$, and Sean C. Smart ${ }^{1}$ \\ ${ }^{I}$ Department of Oncology, CRUK/MRC Gray Institute for Radiation Oncology and Biology, University of Oxford, Oxford, United \\ Kingdom; and 2Department of Radiology, UZ Brussel, Laarbeeklaan 101/103, B-1090, Jette, Belgium
}

\begin{abstract}
CT is widely used for anatomic referencing of PET and SPECT images of small animals but requires sufficiently high radiation doses capable of causing significant DNA damage. Therefore, we described the relationship between radiation dose, biologic damage, and image quality to determine whether CT can be used without significantly compromising radiotherapy and tumor development studies. Methods: The CT dose index generated by the nanoSPECT/CT system was compared with measurements using EBT2 gafchromic film. The effects of micro-CT were evaluated in 2 mouse strains that differ in sensitivity to radiation. $\gamma \mathrm{H} 2 \mathrm{AX}$ foci analysis to determine leukocyte, liver, and jejunum DNA damage and hematoxylin and eosin staining to investigate macroscopic jejunum damage were performed. Signal-to-noise ratio, contrast-to-noise ratio, and scanner linearity were determined to assess image quality. Results: For the standard settings, that is, as set by the manufacturers, EBT2 gafchromic film dosimetry showed that the nanoSPECT/ CT system underestimated the absorbed dose. Moreover, significant doses were obtained, resulting in a significant increase in $\gamma \mathrm{H} 2 \mathrm{AX}$ formation in leukocytes, liver, and jejunum $40 \mathrm{~min}$ after CT, using preset parameters when compared with nonimaged controls. The jejenum response was more pronounced for the more radiosensitive strain. In contrast to leukocytes, the liver and jejunum still showed evidence of DNA damage $3 \mathrm{~d}$ after CT. Contrast-to-noise ratio, signal-to-noise ratio, and scanner linearity were sufficient to allow for anatomic referencing for both imaging protocols tested. Conclusion: Anatomic reference images can be produced with no observable DNA damage or compromising image quality using low radiographic voltage, flux, and duration.
\end{abstract}

Key Words: micro-CT; radiation dose; biologic damage

J Nucl Med 2011; 52:1827-1833

DOI: 10.2967/jnumed.111.089151

$\mathbf{T}$ he abundance of transgenic mouse models has led to a better understanding of the underlying genetic and molecular alterations in cancer that can be well characterized through

\footnotetext{
Received Feb. 11, 2010; revision accepted Jul. 29, 2011.

For correspondence or reprints contact: Veerle Kersemans, Old Road Campus Research Building (off Roosevelt Dr.), Oxford, OX3-7DQ, U.K.

E-mail: Veerle.Kersemans@oncology.ox.ac.uk

Published online Oct. 7, 2011.

COPYRIGHT @ 2011 by the Society of Nuclear Medicine, Inc.
}

the use of noninvasive small-animal imaging techniques. Both PET and SPECT are capable of measuring the biodistribution of picomolar concentrations of radiolabeled biomolecules in small animals and of quantifying the molecular processes in which they participate. However, because no single imaging modality can provide all informationstructural, functional, and molecular-in 1 image, PET or SPECT is often combined with CT. This dual-modality setup greatly facilitates regional anatomic localization of focally increased radiopharmaceutical uptake.

Today, CT is widely used for anatomic referencing of PET and SPECT images of small animals. However, when standard settings as provided by the manufacturers are used, relatively high radiation doses may be delivered to the animal to achieve good image quality. Although these are in general sublethal, they may be sufficient to induce significant DNA damage and thus confound experimental outcome, especially in a radiobiology research study (1). Therefore, dose accumulation must be considered and efforts have to be increased to optimize micro-CT settings to minimize the dose delivered while still maintaining sufficient image quality.

In this article, we describe the relationship between radiation dose, radiation-induced DNA double-strand breaks (DSB), and image quality and show that anatomic referencing can be accomplished without causing prolonged DNA damage or compromising the accuracy of hotspot localization when reducing the recommended manufacturer's settings for radiographic voltage, flux, and duration.

\section{MATERIALS AND METHODS}

\section{System}

The CT component of the Bioscan nanoSPECT/CT system was used. The cone-beam $\mathrm{x}$-ray source with a $90-\mathrm{kVp}$ microfocus tube (focal spot size, $18 \mu \mathrm{m}$ ) was operated in spiral scan mode. The detector contained a 1,024 $\times 2,048$ pixel photodiode array with $48-\mu \mathrm{m}$ pixel spacing. The geometric magnification was 1.3 , and the nominal beam width at isocenter was $50 \mathrm{~mm}$. Details of the imaging parameters are presented in Table 1 (settings used for dosimetry) and Table 2 (settings to study biologic damage).

The manufacturer's calculated CT dose index (CTDI) represents the average absorbed dose, along the $z$-axis, from a series of contiguous irradiations. The CTDI was based on the integration 
TABLE 1

Variation in Absorbed Dose in Mouse Phantom from CT Scan for a Range of Imaging Parameters

\begin{tabular}{|c|c|c|c|c|c|}
\hline Parameter & $\begin{array}{c}35 \mathrm{kV}, \\
50 \mu \mathrm{A}, 400 \mathrm{~ms}, \\
180 \text { projections, } \\
\text { CT1 }\end{array}$ & $\begin{array}{c}45 \mathrm{kV}, \\
177 \mu \mathrm{A}, 1,600 \mathrm{~ms}, \\
180 \text { projections }{ }^{*} \\
\text { CT2 }\end{array}$ & $\begin{array}{c}55 \mathrm{kV}, \\
145 \mu \mathrm{A}, 500 \mathrm{~ms}, \\
180 \text { projections*, } \\
\text { CT3 }\end{array}$ & $\begin{array}{c}65 \mathrm{kV}, 123 \mu \mathrm{A}, \\
500 \mathrm{~ms}, \\
180 \text { projections*, } \\
\text { CT4 }\end{array}$ & $\begin{array}{c}65 \mathrm{kV}, \\
123 \mu \mathrm{A}, 2,000 \mathrm{~ms}, \\
360 \text { projections, } \\
\text { CT5 }\end{array}$ \\
\hline Skin dose & 0.013 & 0.22 & 0.079 & 0.087 & 0.69 \\
\hline Center dose & 0.006 & 0.11 & 0.043 & 0.050 & 0.41 \\
\hline Weighted average & 0.0083 & 0.15 & 0.056 & 0.064 & 0.53 \\
\hline CTDI & 0.028 & 0.096 & 0.035 & 0.041 & 0.331 \\
\hline
\end{tabular}

*Standard imaging settings as provided by manufacturer.

Data are in grays. Dose for given radial distribution is average value across length of phantom. Skin dose represents average dose in surface layer to depth of $0.5 \mathrm{~mm}$, center dose is average dose to cylinder of radial distance of $0.5 \mathrm{~mm}$ from center, and weighted average is average dose to phantom.

of the radiation dose profile from a single axial scan over \pm 50 $\mathrm{mm}$, corresponding to the $100-\mathrm{mm}$ length of a pencil ionization chamber within a 60 -mm-diameter cylindric polymethyl methacrylate phantom.

\section{Dosimetry}

The variation in dose per CT scan and associated dose distributions for a range of imaging parameters was performed using EBT2 gafchromic film (ISP Technologies Inc.), which has a low energy dependence, with a $6.5 \%$ variation in optical density to absorbed dose response for x-ray beam irradiations with energies ranging from $50 \mathrm{kVp}$ up to $10 \mathrm{MV}(2)$. The film $(70 \times 24.5 \mathrm{~mm})$ was sandwiched between 2 identical semicylinders of WT1 waterequivalent material (Barts and the London NHS Trust), which made up a cylindric mouse phantom (length, $70 \mathrm{~mm}$; diameter, $25.4 \mathrm{~mm}$ ). The phantom was positioned central to the CT scanner, with its axis parallel to the axis of the scanner and the film aligned in the horizontal plane. The phantom was imaged across the entire length, and the number of scans per film exposure for a given set of imaging parameters was varied to ensure sufficient exposure of the film to facilitate accurate analysis. The exposed film strips were processed using an Epson-10000XL scanner at least $24 \mathrm{~h}$ after exposure. Optical density values were corrected as recommended by the manufacturer and converted to dose values using a previously obtained calibration curve. The dose per CT scan (skin dose, center dose, and weighted average) was determined by dividing the number of scans used during exposure of the film. The latter dose was compared with the CTDI as calculated by the
nanoSPECT/CT system. In addition, the standard CT settings as set by the manufacturer were altered to increase or reduce the absorbed dose to the animal or phantom (CT protocols CT1 and CT5, Table 1).

\section{Animals}

Animal studies were performed in accordance with the Animals Scientific Procedures Act of 1986 (U.K.). The effects of micro-CT were evaluated in 2 mouse strains that differed in sensitivity to radiation: $\mathrm{C} 57 \mathrm{BL} / 6$ (resistant) and $\mathrm{BALB} / \mathrm{c}$ severe combined immune-deficient (SCID) (sensitive; defective in DNA repair). Scoring sheets assessing mobility, reactivity to handling, coat appearance, and weight loss were used to identify macroscopic distress in mice after micro-CT. All mice (Charles River; $n=5 /$ group) were anesthetized using $2 \%$ isoflurane in oxygen.

\section{Leukocyte DNA Damage}

Six different CT protocols (CT1, CT2, CT6, CT7, CT8, and CT9; Table 2) were applied, and the results were compared with nonirradiated mice. Although CT8 and CT9 are less relevant in preclinical experimentation, as multiple scans were needed, they were used to define the dose-response relationship. At $40 \mathrm{~min}$ or $24 \mathrm{~h}$ after irradiation, mice were sacrificed, and whole blood was collected in an ethylenediaminetetraacetic acid-coated vacutainer (VWR). $\gamma$-H2AX staining, based on the method as described by Rothkamm et al. and described in the supplemental data (supplemental materials are available online only at http://jnm. snmjournals.org), was performed. The percentage of positive

TABLE 2

Micro-CT Parameters to Achieve Average Absorbed Doses as Measured by EBT2 Film and Reported in Figures 1 and 2

\begin{tabular}{|c|c|c|c|c|c|c|}
\hline Protocol & $\begin{array}{l}\text { Tube potential } \\
\qquad(\mathrm{kV})\end{array}$ & $\begin{array}{c}\text { Tube current } \\
(\mu \mathrm{A})\end{array}$ & $\begin{array}{l}\text { Exposure time } \\
\text { (ms) }\end{array}$ & $\begin{array}{c}\text { No. of } \\
\text { projections }\end{array}$ & $\begin{array}{l}\text { No. of } \\
\text { acquisitions }\end{array}$ & $\begin{array}{c}\text { Average dose } \\
\text { (mGy) }\end{array}$ \\
\hline CT1 & 35 & 50 & 400 & 180 & 1 & 8.3 \\
\hline CT2 & 45 & 177 & 1,600 & 180 & 1 & 150 \\
\hline CT6 & 45 & 177 & 2,000 & 360 & 1 & 424 \\
\hline CT7 & 65 & 123 & 1,600 & 360 & 1 & 562 \\
\hline CT8 & 45 & 177 & 2,000 & 360 & 2 & 1,125 \\
\hline CT9 & 55 & 145 & 2,400 & 360 & 3 & 2,419 \\
\hline CT10 & 65 & 123 & 2,400 & 360 & 4 & 3,361 \\
\hline
\end{tabular}


$\gamma \mathrm{H} 2 \mathrm{AX}$ cells was determined using flow cytometry, and data were normalized for control staining (3).

\section{Liver and Jejunum DNA Damage}

The liver and jejunum, classified by Rubin and Casarett as being of fairly low radiosensitivity and highly radiosensitive, respectively, were chosen for further examination (4). Three different CT protocols (CT1, CT7, and CT10; Table 2) were tested, and the results were compared with nonirradiated mice. CT10 served as a positive control. At $40 \mathrm{~min}$ or $72 \mathrm{~h}$ after irradiation, mice were sacrificed. Liver and jejunum were dissected and stained for $\gamma \mathrm{H} 2 \mathrm{AX}$ (supplemental data). The number of $\gamma \mathrm{H} 2 \mathrm{AX}$ foci per nucleus was counted for a minimum of 100 cells per condition.

\section{Macroscopic Jejunum Damage}

Jejunum was prepared as described above, before hematoxylin and eosin staining (supplemental data).

\section{Image Quality}

The quality assurance phantom developed by Eloot et al. was used to assess the image quality (5). Imaging settings CT1 and CT5 were applied, and contrast-to-noise (CNR), signal-to-noise (SNR), and scanner linearity were determined using ImageJ (6). The vials of the contrast section were filled with concentrations of Iomeron-400 (Bracco U.K. Ltd.), an iodinated contrast agent, ranging from 4.0 to $40.0 \mathrm{mg}$ of iodine per milliliter, or with water.

\section{Statistics}

Leukocyte, liver, and jejunum DNA damage were compared for different micro-CT parameters by 1-way parametric ANOVA using Tukey adjustment for multiple comparisons $(P<0.05)$. The relationship between signal intensity and iodine concentration was determined by linear regression analysis. All statistical tests were performed in Prism 5 (GraphPad Software).

\section{RESULTS}

The radial variation in dose per scan across the mouse phantom for a range of imaging parameters is shown in Supplemental Figure 1. The dose represents the average value across the length phantom. These data were used to calculate the skin dose (average dose in the surface layer to a depth of $0.5 \mathrm{~mm}$ ), the center dose (average dose to a cylinder of radial distance of $0.5 \mathrm{~mm}$ ), and the weighted average dose (average dose to the phantom) and are presented in Table 1 along with the CTDI as calculated by the SPECT/CT system. The values illustrate the significant variation in dose received as a function of imaging parameters used. For the standard settings, the CTDI underestimated the absorbed dose by 2.3-, 1.2-, and 1.6-fold for the skin dose, center dose, and weighted average dose, respectively. However, when a softer x-ray beam was used in an effort to lower the radiation dose, the manufacturer's CTDI value was significantly higher than our data. The radial distribution of dose is significantly influenced by the tube voltage used for imaging; the ratio of surface-tocenter dose increases with decreasing $\mathrm{kV}$ because of the increased attenuation of the low-energy component of the $\mathrm{x}$-ray beam through the mouse phantom. The radial variation in dose presented in Supplemental Figure 1 is slightly asymmetric as a result of inaccuracies in positioning the phantom central to the scanner axis. Additionally, there is a variation in dose along the $z$-axis of the phantom, as shown in Supplemental Figure 2. The dose falls off toward the edges of the phantom. In the center of the phantom, there is significant contribution to the dose from radiation scattered from the surrounding material, whereas at the edges of the phantom the contribution of scattered radiation falls because of minimal scatter from air. The dose distribution along the $z$-axis is also perturbed because of repositioning the table during the scan to enable scans more than $36 \mathrm{~mm}$, resulting in additional contribution of dose in the overlapping region.

$\gamma \mathrm{H} 2 \mathrm{AX}$ foci analysis was performed on leukocytes of both C57BL/6 and BALB/c SCID mice at $40 \mathrm{~min}$ and 24 $\mathrm{h}$ after irradiation. As presented in Figures $1 \mathrm{~A}$ and 2A, a linear radiation dose-response relationship was obtained for both mouse strains when a range of radiographic settings was applied. Moreover, at the same time point after irradiation, BALB/c SCID mice were less able to repair DNA DSBs, as evidenced by the steeper slope. Only at the lowest radiographic setting (CT1) was no significant

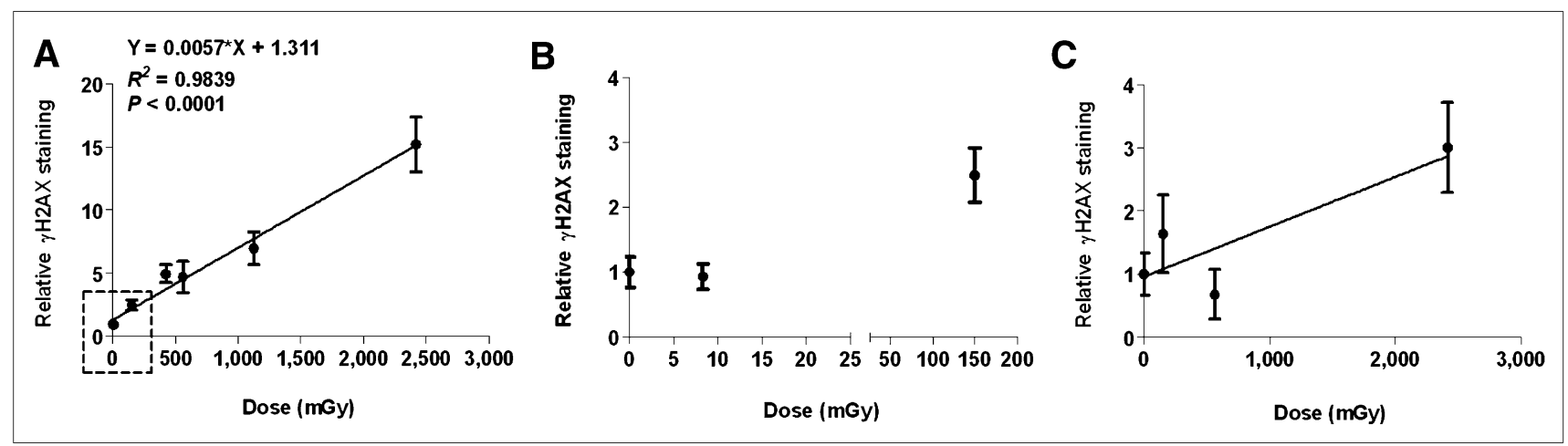

FIGURE 1. $\gamma \mathrm{H} 2 \mathrm{AX}$ foci analysis on leukocytes of C57BL/6 mice at $40 \mathrm{~min}(\mathrm{~A}$ and $\mathrm{B}$ ) and $24 \mathrm{~h}(\mathrm{C})$ after irradiation. (B) Results at lower doses as indicated by box in A. Reported measured average absorbed doses resulted from imaging parameters as described in Table 2 . Results are expressed as mean \pm SD and as function of average absorbed dose measured by EBT2 film. 


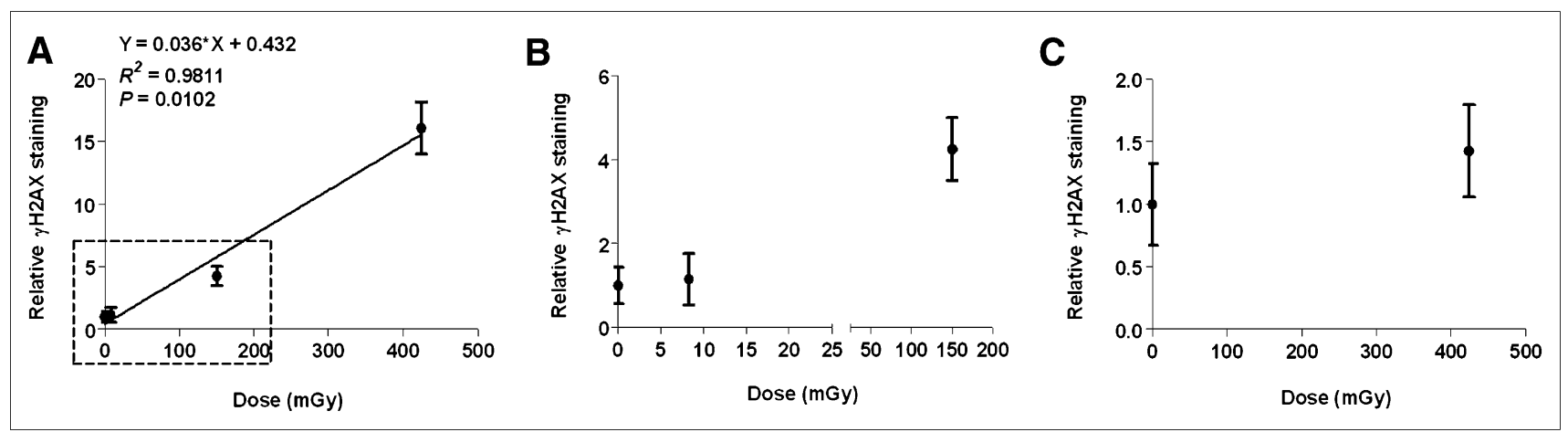

FIGURE 2. $\gamma \mathrm{H} 2 \mathrm{AX}$ foci analysis on leukocytes of BALB/c SCID mice at $40 \mathrm{~min}(\mathrm{~A}$ and $\mathrm{B})$ and $24 \mathrm{~h}(\mathrm{C})$ after irradiation. (B) Results at lower doses as indicated by box in A. Reported measured average absorbed doses resulted from imaging parameters as described in Table 2. Results are expressed as mean \pm SD and as function of average absorbed dose measured by EBT2 film.

increase in DNA damage observed for either mouse strains. Likewise, $\gamma \mathrm{H} 2 \mathrm{AX}$ foci formation was studied in 2 additional organs, that is, the liver and jejunum, which differ in their relative sensitivity to radiation. The results are summarized in Table 3 and are similar to those observed in leukocytes. Again, at $40 \mathrm{~min}$ after irradiation, no differences in $\gamma \mathrm{H} 2 \mathrm{AX}$ foci formation were observed for CT1 for either organ $(P=0.46$ and 0.76 for liver and jejunum, respectively). However, for CT7, the results diverge. Although DNA damage in leukocytes and jejunum was obvious, this was not so for the liver $(P=0.05)$. As expected, a huge increase in $\gamma \mathrm{H} 2 \mathrm{AX}$ foci count was detected for the positive control, CT10, for both organs.

Although the acute effects of micro-CT were clear, no significant differences in $\gamma \mathrm{H} 2 \mathrm{AX}$ staining in leukocytes were detected between control and irradiated mice at $24 \mathrm{~h}$ after irradiation, as shown in Figures $1 \mathrm{C}$ and $2 \mathrm{C}$. The latter was in contrast to liver and jejunum, which still showed an increase in foci at $3 \mathrm{~d}$ after irradiation for CT7 $(P<0.001$ for both organs) but not CT10 $(P=0.04$ and 0.12 for jejunum and liver, respectively).

Macroscopic changes after micro-CT were monitored using scoring sheets, but no obvious signs of radiation sickness were observed for any group except for CT10. The radiographic settings for $\mathrm{CT} 10$ resulted in $18 \% \pm 3 \%$ weight loss, a general lack of grooming, and less mobility as compared with controls at $3 \mathrm{~d}$ after irradiation. Therefore, to avoid further distress to the animals, the mice were culled and the endpoint of the study was reached. For CT1, CT7, and CT10, the macroscopic appearance of the jejunum was studied at $40 \mathrm{~min}$ and $3 \mathrm{~d}$ after irradiation, and these results are presented in Figure 3. For all tested conditions, except for CT10 at $3 \mathrm{~d}$, the light micrograph showed intact, regular, long fingerlike villi with crypts of Lieberkühn at their bases. However, the positive control, CT10, displayed short, irregular, distorted villi with numerous vacuoles replacing the crypts of Lieberkühn.

CNR, SNR, and scanner linearity were determined to assess image quality for the high-resolution standard setting CT5 and the adapted setting CT1. A summary of the obtained CNRs and SNRs is presented in Table 4. The scanner linearity as tested with the contrast section of the phantom was good, with $R^{2}$ values of 0.9945 and 0.9975 for CT1 and CT5, respectively. Representative images of the resolution section are shown in Figure 4.

\section{DISCUSSION}

When planning small-animal imaging experiments, especially when radiobiology and radiotherapy are involved, optimization of the CT protocol is strongly advised, to minimize the biologic consequences of the additional dose associated with the CT while maintaining sufficient resolution. The literature shows that researchers most often use preset parameters, defined by the manufac-

TABLE 3

$\gamma \mathrm{H} 2 \mathrm{AX}$ Foci Formation in Liver and Jejunum of BALB/c SCID mice at 40 Minutes and 72 Hours After Irradiation

\begin{tabular}{|c|c|c|c|c|}
\hline \multirow[b]{2}{*}{ Parameter } & \multicolumn{2}{|c|}{ Liver } & \multicolumn{2}{|c|}{ Jejunum } \\
\hline & 40 min after irradiation & $72 \mathrm{~h}$ after irradiation & 40 min after irradiation & $72 \mathrm{~h}$ after irradiation \\
\hline No irradiation (control) & $0.46 \pm 0.10$ & $0.12 \pm 0.05$ & $0.76 \pm 0.11$ & $0.09 \pm 0.04$ \\
\hline CT1 & $0.66 \pm 0.05$ & $0.18 \pm 0.05$ & $0.86 \pm 0.15$ & $0.11 \pm 0.04$ \\
\hline CT7 & $1.85 \pm 0.53$ & $0.50 \pm 0.05$ & $3.26 \pm 0.23$ & $0.55 \pm 0.01$ \\
\hline CT10 & $11.45 \pm 0.44$ & $0.05 \pm 0.03$ & $15.48 \pm 0.30$ & $0.22 \pm 0.09$ \\
\hline \multicolumn{5}{|c|}{$f$ foci per nucleus \pm SE } \\
\hline
\end{tabular}




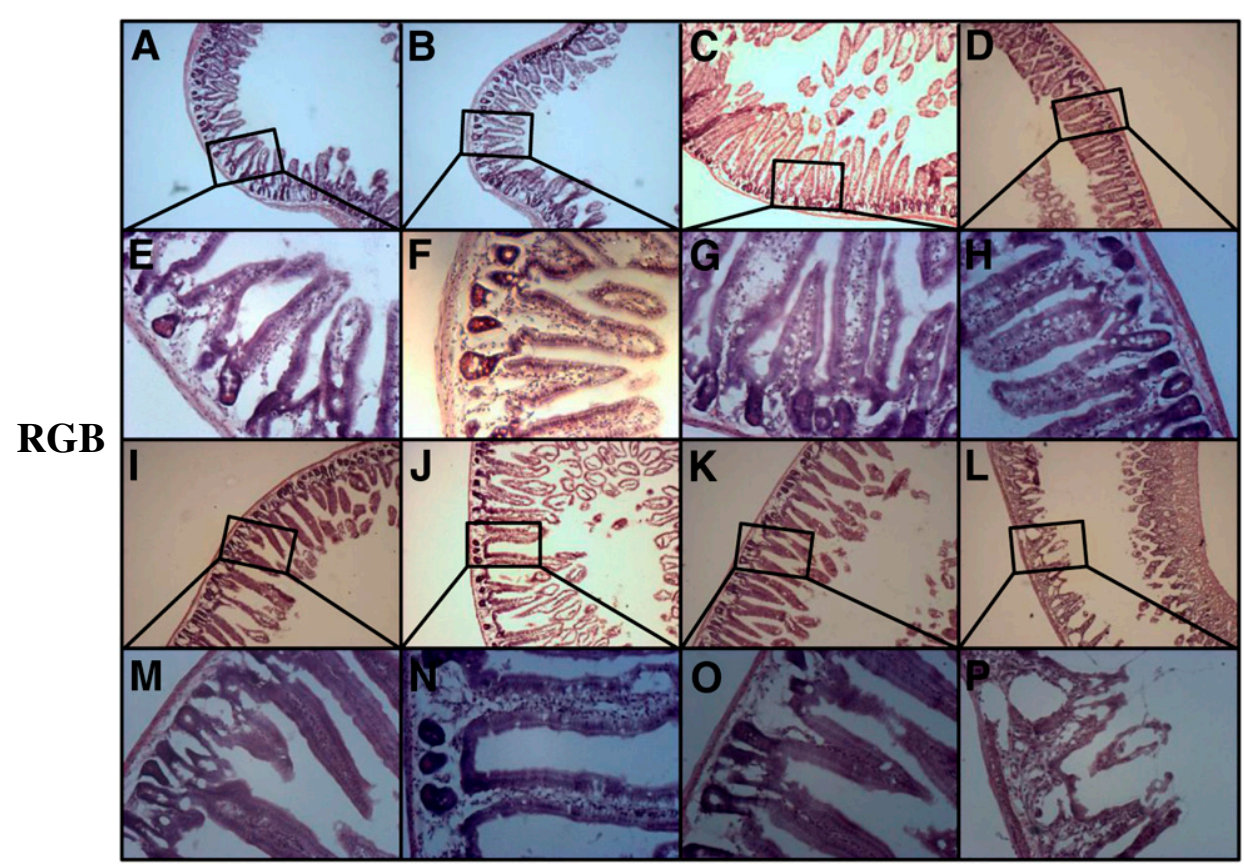

FIGURE 3. Light microgrograph showing effects of micro-CT on jejunum (hematoxylin and eosin stain). (A-D) Magnification ( $\times 2.5$ ) of acute effects at 40 min after irradiation. Shown are magnification $(\times 10)$ of slides A-D $(E-H)$, magnification $(\times 2.5)$ of chronic effects at $3 \mathrm{~d}$ after irradiation (I-L), and magnification $(\times 10)$ of slides $\mathrm{I}-\mathrm{L}(\mathrm{M}-\mathrm{P})$. turers. Although they usually result in good image quality, the radiation dose to the animal can be significant.

Few, often contradictory, studies investigated the effects of $\mathrm{CT}$ on experimental outcomes in small-animal models in vivo (7-10). Carlson et al. (10) and more recently Foster and Ford (8) determined the impact of longitudinal microCT on tumor growth. These authors concluded that the effects of the radiation dose delivered during longitudinal imaging using standard settings did not cause statistically significant differences in final tumor volumes. Opposing those results are those obtained by Laforest et al., who suggested that micro-CT can induce tumor inhibition (9). Furthermore, Willekens et al. (7) demonstrated in normal C57BL/6 mice that the median mouse organ dose resulting from a standard micro-CT scan is $406 \pm 44 \mathrm{mGy}$ - a dose capable of influencing experimental outcome. Although reports exist that micro-CT has the potential to deliver high doses to organs and tumors, the biologic impact has not yet been described. Moreover, measurement of tumor growth might be a too-insensitive parameter to observe biologic effects because more subtle but significant damage that can alter tumor biology might be present before tumor

TABLE 4

Assessment of Image Quality After Micro-CT

\begin{tabular}{crrrrrr}
\hline & \multicolumn{2}{c}{ CT1 } & & \multicolumn{2}{c}{ CT5 } \\
\cline { 2 - 3 } \cline { 6 - 7 } lodine concentration $(\mathrm{mg} / \mathrm{mL})$ & CNR & SNR & & CNR & SNR \\
\hline 4 & 4.09 & 3.76 & & 7.37 & 4.89 \\
8 & 4.57 & 5.78 & & 8.75 & 6.21 \\
20 & 4.95 & 7.61 & & 13.82 & 7.56 \\
40 & 5.23 & 8.26 & & 17.68 & 7.92 \\
\hline
\end{tabular}

shrinkage or stagnation is observed. However, this shortcoming does not matter when tumor volume is the endpoint of the study. On the other hand, studies involving SPECT/ CT or PET/CT often look at changes in molecular processes or microenvironment, and these can be altered dramatically after radiation (11). Thus, more sensitive readouts are essential, and determining the relationship between imaging parameters, image quality, and radiation damage might be a useful parameter for the preclinical imaging community.

In conjunction with radiation damage studies, it was important to characterize the performance of our CT equipment. Therefore, the CTDI as calculated by the manufacturers was compared with measurements of absorbed dose using EBT2 gafchromic film. For the standard settings, this comparison revealed that the CTDI resulted in an underestimation of the absorbed dose but significantly overestimated the absorbed dose when standard settings were altered to image at $35 \mathrm{kV}, 50 \mu \mathrm{A}$. Moreover, significant doses (well into the centigray range) were obtained when the standard settings, that is, as set by the manufacturers, were used (Table 1). Fortunately, the average absorbed dose could be lowered at least 7 -fold by altering the preset parameters as described-a dose that was comparable to earlier published work (7). Moreover, the radial dose distribution illustrated that the $\mathrm{x}$-rays are significantly attenuated as they traverse the body of the mouse (or phantom), typically leading to a relatively higher dose to the skin than to the body or organs situated more centrally. These differences increase with decreasing $\mathrm{x}$-ray energy because of greater attenuation through the body.

Having better knowledge about the true dose delivered by micro-CT facilitates the study of biologic effects 


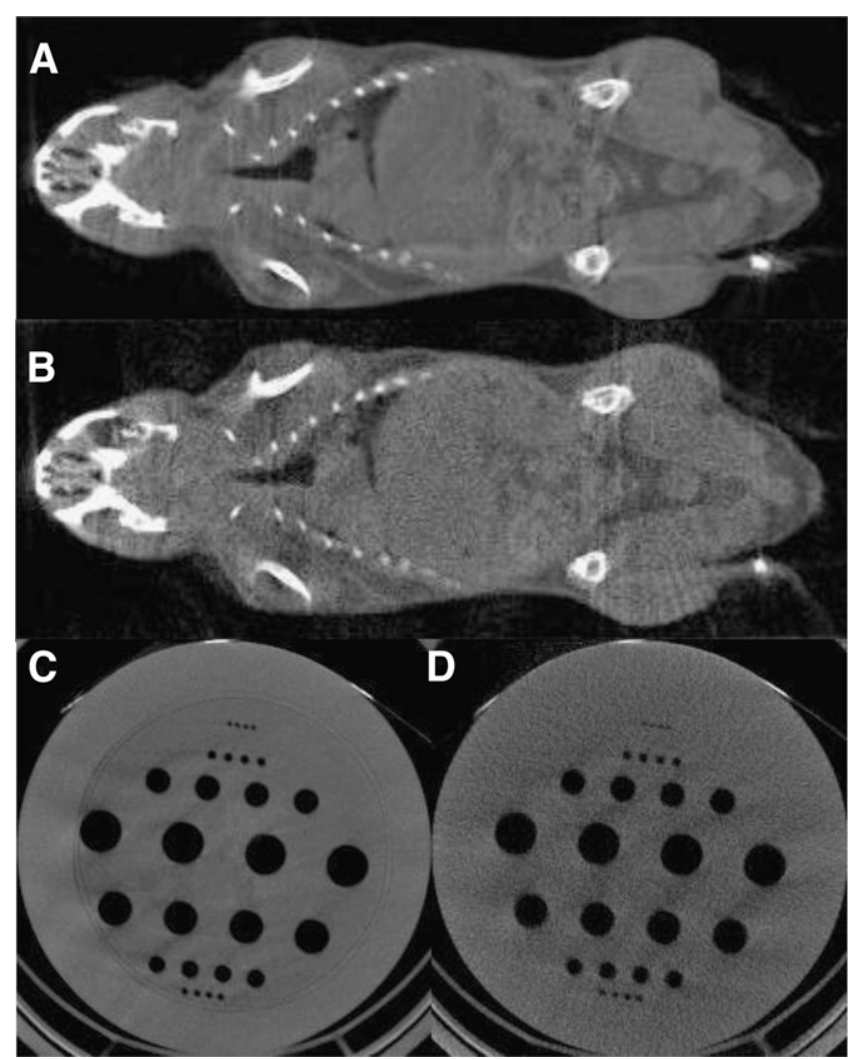

FIGURE 4. Representative high-resolution (A and C, using CT5) and low-resolution ( $B$ and $D$, using $C T 1$ ) micro-CT images of mouse $(A$ and $B)$ and resolution section of phantom ( $C$ and $D)$.

associated with the scan and how they potentially perturb the results of animal experiments. To date, one of the more sensitive assays for determining radiation effects is DNA damage response. DNA DSBs are caused directly by irradiation and some radiomimetic drugs or indirectly through replication fork stalling (12). One of the earliest stages of cellular response to a DNA DSB is the phosphorylation of histone $\mathrm{H} 2 \mathrm{AX}$ and the accumulation of DNA repair proteins. This H2AX histone variant gets phosphorylated on Ser139, starting immediately after DNA DSB formation (13). Foci containing multiple copies of phosphorylated $\mathrm{H} 2 \mathrm{AX}(\gamma \mathrm{H} 2 \mathrm{AX})$ will form and create the basis of a sensitive and widely applied in vitro assay (14). Therefore, we assessed H2AX phosphorylation as a sensitive biologic dosimeter of radiation exposure in leukocytes, liver, and jejunum of mice that underwent CT. A significant increase in $\gamma \mathrm{H} 2 \mathrm{AX}$ foci was observed in leukocytes using preset parameters when compared with nonimaged controls. The sensitivity of the method is demonstrated by the increase in $\gamma \mathrm{H} 2 \mathrm{AX}$ staining for the DNA repair-deficient, more radiosensitive BALB/c SCID strain, as is reflected in the slope of both linear curves (0.191 vs. 0.079 for $\mathrm{BALB} / \mathrm{c}$ SCID and C57BL/6, respectively). More importantly, the results in Figures 1A and 2A clearly illustrate that CT using preset parameters does induce substantial DNA damage that might be sufficient to alter experimental outcome, especially with respect to a radiobiology endpoint $(3,15,16)$. To minimize the biologic consequences of these scans, guidance for lowering the $\mathrm{x}$-ray voltage, flux, and duration settings of the CT acquisition was obtained from the manufacturers. In consequence, the radiation dose delivered to the animal could be decreased in such a way that no significant DNA damage could be detected 40 min after exposure when compared with nonimaged mice. Additionally, $\gamma \mathrm{H} 2 \mathrm{AX}$ foci analysis was performed in 2 abdominal organs that differ in relative sensitivity to radiation: liver is considered to be fairly insensitive whereas blood and intestines are highly radiosensitive (4). Results were similar to those obtained for leukocytes. Whereas CT2, a sequence designed to minimize the biologic effects, did not show any significant differences in $\gamma \mathrm{H} 2 \mathrm{AX}$ foci count when compared with controls, the use of standard imaging parameters as in CT7 did show a difference. However, it was less obvious for liver than for jejunum, again indicating the sensitivity of the assay.

These results all refer to the acute, microscopic effects of radiation caused by CT. However, from an ethical and experimental point of view, it is equally important to know the longer-lasting effects of these radiation-induced DNA damages. Indeed, one of the great advantages of preclinical imaging is the ability to perform longitudinal studies so that a single group of animals can be tracked throughout an entire investigation. Therefore, when CT is included, these studies might be limited by the fact that ionizing radiation is involved, as often daily imaging sessions are required. Thus, minimizing the dose associated with each scan can help minimize the biologic consequences of these scans. Our study indicates that $24 \mathrm{~h}$ after CT using the most severe parameters, no detectable DNA damage could be observed in leukocytes. However, this finding may not reflect the lack of effect of the CT scan on the underlying biology but simply the biologic half-life of $\gamma \mathrm{H} 2 \mathrm{AX}$. As a consequence, DNA DSB or long-term consequences associated with damage produced at the time of the scan still might be present, as was confirmed by the $\gamma \mathrm{H} 2 \mathrm{AX}$ foci analysis in liver and jejunum. When the standard imaging parameters were used (CT7), DNA DSBs still were detectable at $3 \mathrm{~d}$ after irradiation. Although one would have expected to see the same pattern for higher doses, it was not found for the positive control, probably because of to deterioration of the jejunum.

Because $\gamma \mathrm{H} 2 \mathrm{AX}$ foci analysis might be a too early a marker to predict eventual radiation toxicity, a longer-term endpoint was chosen. For this purpose, all mice were monitored daily for signs of radiation sickness, and the macroscopic appearance of the jejunum was studied at $40 \mathrm{~min}$ and $3 \mathrm{~d}$ after imaging. Only the positive control (CT10) displayed signs of radiation sickness-that is, severe weight loss and the inability to move. Moreover, the internal lining of the small intestine was largely destroyed. Thus, although the biologic effects of radiation were obvious when looking 
at DNA DSB, they did not translate into macroscopic changes. Nevertheless, care should be taken when performing longitudinal CT studies because exposure to low radiation doses, which might not lead to macroscopic damage, might lead to additional concerns such as radiation hypersensitivity (16) and adaptive response (17).

Although these changes in acquisition parameters inevitably lead to decreased resolution, CNR, and SNR, image quality was still adequate enough to identify anatomic features. Moreover, SNR values met the Rose criterion, which states that an SNR of at least 5 is needed to distinguish image features at $100 \%$ certainty (18). The results obtained for CNR were comparable to those reported in literature (5).

\section{CONCLUSION}

These studies were designed to demonstrate the damaging effects of micro-CT in small-animal imaging, but more studies are needed if the results are to be extrapolated to preclinical radiotherapy or tumor development studies. In general, awareness of radiographic dosing must be included in the optimization of imaging protocols, because we have shown that standard micro-CT does result in considerable DNA damage. Although these effects were not propagated into a macroscopic setting, the DNA damage was significant and still not fully repaired in liver and jejunum at $3 \mathrm{~d}$ after imaging. Moreover, the cumulative effects of radiation dosing are well established-hence fractionated radiotherapy. Taking into account the linear relationship between dose and leukocyte DNA damage, one can expect an accumulative effect when performing longitudinal studies. Consequently, to lower the radiation burden to the animal, we recommend using low $\mathrm{x}$-ray voltage, flux, and duration and avoiding multiple scans. The altered settings as reported in this article did not generate observable DNA damage and maintained sufficient image quality.

Additionally, we report that the CTDI, when provided by manufacturers, should be used as guidance to the absorbed dose in only the center of the subject. If more accurate values are required throughout the subject, then these should be determined using standard dosimetry methods. It is possible to measure the induction and subsequent repair of DNA damage using flow cytometry to measure $\gamma \mathrm{H} 2 \mathrm{AX}$ in leukocytes of mice undergoing CT scans. By this means, the biologic impact of radiation exposure from a CT scan can be assessed.

\section{DISCLOSURE STATEMENT}

The costs of publication of this article were defrayed in part by the payment of page charges. Therefore, and solely to indicate this fact, this article is hereby marked "advertisement" in accordance with 18 USC section 1734.

\section{ACKNOWLEDGMENTS}

This work was funded by Cancer Research U.K. (C5255/ A12678) and the Oxford Cancer Imaging Centre (C5255/ A10339). No other potential conflict of interest relevant to this article was reported.

\section{REFERENCES}

1. Boone JM, Velazquez O, Cherry SR. Small-animal x-ray dose from micro-CT. Mol Imaging. 2004;3:149-158.

2. Butson MJ, Yua PKN, Cheunga T, Alnawaf H. Energy response of the new EBT2 radiochromic film to X-ray radiation. Radiat Meas. 2010;45:836-839.

3. Rothkamm K, Balroop S, Shekhdar J, Fernie P, Goh V. Leukocyte DNA damage after multi-detector row CT: a quantitative biomarker of low-level radiation exposure. Radiology. 2007;242:244-251.

4. Rubin P, Casarett GW. Clinical radiation pathology as applied to curative radiotherapy. Cancer. 1968;22:767-778.

5. Eloot L, Buls N, Covens P, Willekens I, Lahoutte T, de Mey J. Quality control of micro-computed tomography systems. Radiat Prot Dosimetry. 2010;139:463-467.

6. Abramoff MD, Magelhaes PJ, Ram SJ. Image processing with ImageJ. Biophotonics Int. 2004;11:36-42.

7. Willekens I, Buls N, Lahoutte T, et al. Evaluation of the radiation dose in microCT with optimization of the scan protocol. Contrast Media Mol Imaging. 2010;5:201-207.

8. Foster WK, Ford NL. Investigating the effect of longitudinal micro-CT imaging on tumour growth in mice. Physics Med Biol. 2011;56:315-326.

9. Laforest R, Waterson CE, Broski SM, Lewis JS. Radiation dose considerations in tumour growth monitoring with CT. Mol Imaging. 2004;3:288.

10. Carlson SK, Classic KL, Bender CE, Russell SJ. Small animal absorbed radiation dose from serial micro-computed tomography imaging. Mol Imaging Biol. 2007;9:78-82.

11. Prise KM, Schettino G, Folkard M, Held KD. New insights on cell death from radiation exposure. Lancet Oncol. 2005;6:520-528.

12. Helleday T, Petermann E, Lundin C, Hodgson B, Sharma RA. DNA repair pathways as targets for cancer therapy. Natl Rev. 2008;8:193-204.

13. Banath JP, Olive PL. Expression of phosphorylated histone $\mathrm{H} 2 \mathrm{AX}$ as a surrogate of cell killing by drugs that create DNA double-strand breaks. Cancer Res. 2003;63:4347-4350.

14. Bonner WM, Redon CE, Dickey JS, et al. GammaH2AX and cancer. Natl Rev. 2008;8:957-967.

15. Joiner MC, Marples B, Lambin P, Short SC, Turesson I. Low-dose hypersensitivity: current status and possible mechanisms. Int J Radiat Oncol Biol Phys. 2001;49:379-389.

16. Longo JA, Nevaldine B, Longo SL, Winfield JA, Hahn PJ. An assay for quantifying DNA double-strand break repair that is suitable for small numbers of unlabeled cells. Radiat Res. 1997;147:35-40.

17. Prise KM. New advances in radiation biology. Occup Med (Lond). 2006;56: $156-161$.

18. Bushberg J, Seibert J, Leidthold E, Boone J. The Essential Physics of Medical Imaging. 2nd ed. Baltimore, MD: Lippincott, Williams \& Wilkins; 2002. 